\title{
$\phi$ DECAY IN NUCLEI
}

\author{
E. Oset ${ }^{1}$ and A. Ramos ${ }^{2}$ \\ 1 Departamento de Física Teórica and IFIC, \\ Centro Mixto Universidad de Valencia-CSIC, \\ Institutos de Investigación de Paterna, Apdo. correos 2085, \\ 46071 Valencia, Spain \\ 2 Departament d'Estructura i Constituents de la Matèria, Universitat de Barcelona, \\ Diagonal 647, 08028 Barcelona, Spain
}

\begin{abstract}
We have studied the decay of the $\phi$ meson in nuclear matter by taking into account the renormalization of the $K$ and $\bar{K}$ mesons in the medium. We separate the contribution of the total width into different reaction channels, $K \bar{K}$ and $K Y h$, the latter ones associated with the $\phi N \rightarrow K^{+} Y$ reactions. We find a total width at normal nuclear matter density of about $22 \mathrm{MeV}$, considerably larger than the free one.
\end{abstract}

PACS: 13.15.-k, 13.75.Jz, 14.40.Ev, 25.75.-q

Keywords: $\phi$ decay in nuclei, Chiral Lagrangian, $\bar{K}$-nucleus interaction 


\section{Introduction}

The renormalization of the hadron properties in a nuclear medium is the object of continuous attentions. Particularly, the properties of the $\rho$ meson in nuclei have been thoroughly studied [1-4] and many experiments are devoted to observe these modifications [5, 6]. Comparatively, the $\phi$ meson has received little attention but the studies of Refs. [4, [7] predict a very small shift, if any, and a substantially increased width. Yet, the study of the $\phi$ width in a nuclear medium is a very interesting question since it should offer information on the renormalization of the kaon properties in a medium, a subject itself which attracts much interest [8 11] and which is related to the possible existence of kaon condensates in neutron stars [12].

The many body problem of the interaction of the $\bar{K}$ with a nuclear medium is a subtle one. The low density theorem leads to a repulsive $\bar{K}$ selfenergy, but analysis of kaonic atoms demands an attractive one 13 [15. A step forward in the understanding of this peculiar feature is given in Refs. [8,9] where it is shown that the Pauli blocking of intermediate states in the $\bar{K} N$ interaction leads to a shift of the $\Lambda(1405)$ resonance (which lies just below the $\bar{K} N$ threshold) to higher energies. As a consequence the $\bar{K} N$ interaction becomes attractive. On the other hand, the attraction felt by the kaons has an opposite effect because it brings the resonance back to lower energies. This fact stimulated a selfconsistent calculation in Ref. [10] which showed that the position of the $\Lambda(1405)$ resonance was not changed in the medium but altogether the $\bar{K}$ still felt an attraction and the $\Lambda(1405)$ resonance broadened considerably. The calculations of Ref. [11] introduced in addition the renormalization of the pions and the baryons in the intermediate states, and found again that the position of the resonance barely changed when the $\bar{K}$ selfenergy was calculated selfconsistently while the width became even larger. The $\bar{K}$ nucleus potential of Ref. [11] has been used in the study of Ref. [16] where it was found to be compatible with the present data of kaonic atoms.

The important changes found in the kaon selfenergy in the medium, which is the key ingredient here, advise a reevaluation of the $\phi$ selfenergy and this is the purpose of the present work. The framework for the evaluation of the $\phi$ selfenergy in a nuclear medium was developed in Ref. [曰, 7] and we will adhere to it, yet following a different technical approach.

\section{$2 \phi$ decay in the nuclear medium}

We will use here the gauge vector representation of the vector field $\phi$. The alternative tensor formulation of Ref. [17] is equivalent to the gauge vector one but makes the chiral counting easier. Yet, in the present problem one needs Lagrangians for the coupling of the mesons to baryons which are available in the vector representation but not in the tensor one and hence 
we shall adhere to the vector representation as done in Refs. [4, 团.

In free space the $\phi$ meson decays into $K \bar{K}$ and $3 \pi$. The latter channel is OZI forbidden and in spite of the large phase space only accounts for $15 \%$ of the total width. The coupling of the $\phi$ to $K \bar{K}$ is large and it is only the reduced phase space what makes the $\phi$ width small. However, we shall see that thanks to the $\bar{K}$ related channels in the medium, the $\phi$ width becomes of the order of $22 \mathrm{MeV}$ at normal nuclear matter density $\left(\rho_{0}=0.17 \mathrm{fm}^{-3}\right)$ and the $3 \pi$ channel represents only $3 \%$ of this width. Thus, we shall neglet it in our study.

The Lagrangian that describes the coupling of the $\phi$ meson to kaons is given by

$$
\mathcal{L}=-i g_{\phi} \phi_{\mu}\left(K^{-} \partial_{\mu} K^{+}-K^{+} \partial_{\mu} K^{-}+\bar{K}^{0} \partial_{\mu} K^{0}-K^{0} \partial_{\mu} \bar{K}^{0}\right),
$$

which provides the following $\phi K^{+} K^{-}$vertex (matrix element of $i \mathcal{L}$ )

$$
V_{\phi K^{+} K^{-}}=-i g_{\phi} \epsilon_{\mu}\left(p^{\mu}-p^{\prime \mu}\right),
$$

with $p, p^{\prime}$ the momenta of the $K^{+}, K^{-}$mesons, respectively. An analogous vertex in found for $K^{0} \bar{K}^{0}$.

We shall work in a frame where the $\phi$ is at rest and in a gauge where $\epsilon^{0}=0$. If we consider the $\phi$ selfenergy diagram of Fig. [1] with $K^{+} K^{-}$as intermediate states we obtain

$$
\Pi_{\phi}=i \int \frac{d^{4} q}{(2 \pi)^{4}} \frac{1}{q^{2}-m_{K}^{2}+i \varepsilon} \frac{1}{(P-q)^{2}-m_{K}^{2}+i \varepsilon} g_{\phi}^{2} \epsilon_{i} \epsilon_{j} 2 q_{i} 2 q_{j},
$$

where $P$ is the fourmomentum of the $\phi$. The imaginary part of $\Pi$ is readily evaluated using Cutkosky rules

$$
\begin{aligned}
\Pi_{\phi} & \rightarrow 2 i \operatorname{Im} \Pi_{\phi} \\
D(q) & \rightarrow 2 i \theta\left(q^{0}\right) \operatorname{Im} D(q) \\
D(P-q) & \rightarrow 2 i \theta\left(P^{0}-q^{0}\right) \operatorname{Im} D(P-q),
\end{aligned}
$$

where $D(q)$ is the kaon propagator. Then, using the relationship

$$
\Gamma_{\phi}=-\frac{\operatorname{Im} \Pi_{\phi}}{M_{\phi}}
$$

with $M_{\phi}$ the $\phi$ mass, we obtain the free width of the $\phi$ for $K^{+} K^{-}$decay

$$
\Gamma_{\phi}=\frac{2}{3} \frac{1}{4 \pi} g_{\phi}^{2} \frac{p^{3}}{M_{\phi}^{2}},
$$

which requires the value $g_{\phi}=4.57$ to obtain agreement with the experimental value of 2.18 $\mathrm{MeV}$ [18]. 


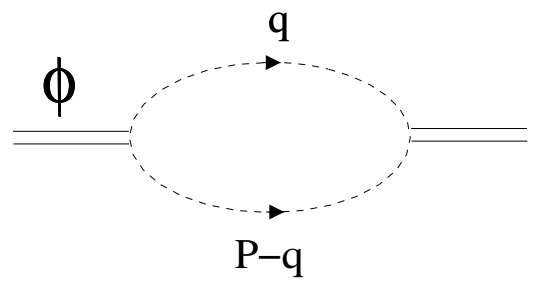

Figure 1: Selfenergy diagram contributing to the decay of the $\phi$ into $K^{+} K^{-}$and $K^{0} \bar{K}^{0}$ pairs.

In the nuclear medium the kaon acquires a selfenergy, $\Pi_{K}\left(q^{0}, \vec{q}, \rho\right)$, and the kaon propagator reads

$$
D(q, \rho)=\frac{1}{q^{02}-\vec{q}^{2}-m_{K}^{2}-\Pi_{K}\left(q^{0}, \vec{q}, \rho\right)},
$$

which can also be cast in terms of the Lehmann representation as

$$
D(q, \rho)=\int_{0}^{\infty} d \omega 2 \omega \frac{S_{K}(\omega, \vec{q}, \rho)}{q^{02}-\omega^{2}+i \varepsilon}
$$

where $S_{K}$ is the kaon spectral function

$$
S_{K}(p, \rho)=-\frac{1}{\pi} \operatorname{Im} D(p, \rho) .
$$

At this point it is worth differentiating between the $\bar{K} \equiv\left(\bar{K}^{0},-K^{-}\right)$and the $K \equiv\left(K^{+}, K^{0}\right)$ meson doublets.

The $K^{+} N$ and $K^{0} N$ interactions are smooth, there are no resonances with strangeness $S=1$ and the selfenergy of the $K^{+}$meson at small energies is well aproximated by the $t \rho$ approximation, which from Refs. [19,20] is given by

$$
\Pi_{K^{+}}=\frac{1}{2}\left(t_{K^{+} p}+t_{K^{+} n}\right) \rho_{0} \frac{\rho}{\rho_{0}}=0.13 m_{K}^{2} \frac{\rho}{\rho_{0}} .
$$

An identical expression is found for the $K^{0}$ meson.

The $\bar{K}$ case is far more subtle. As we pointed out, the $\bar{K} N$ interaction at low energies is dominated by the $\Lambda(1405)$ resonance which appears just below the $\bar{K} N$ threshold. Any realistic approach to the problem has do deal properly with this resonance, which is actually one of the resonances which meets with more problems within quark models of baryons. Not surprisingly, the resonance is closely tied to the interaction of the $\bar{K} N$ system with related channels and is generated within unitary coupled-channel schemes [21 23]. An important step forward has been given with the use of chiral Lagrangians within a coupled channel unitary approach [19,20], by means of which one is able to reproduce the $\Lambda(1405)$ resonance and the cross sections for $K^{-} p$ scattering to the different coupled channels. This succesful scheme is most appropriate to undertake the study of the kaon selfenergy in a nuclear medium and this 
was done in Refs. [9 11]. As quoted above, with respect to the work of Ref. [9], the work of Ref. [10] implemented the selfenergy of the kaon in the calculation in a selfconsistent way, with the important finding that the position of the $\Lambda(1405)$ resonance was barely moved and the $K^{-}$experienced a moderate attraction. The work of Ref. [11 improves on the one of Ref. [10] by including the selfenergy of the pions and the baryons in the intermediate states, thus opening more channels for reactions of the $K^{-}$in the medium, consequently increasing the width of the $\Lambda(1405)$ resonance and widening considerably the spectral function of the $K^{-}$(see also Ref. [24] for a recent review on this and related issues). Clearly, the spread of the kaon strength necessarily should have repercussion on the $\phi$ decay in the medium since it automatically enlarges the phase space for the $\phi$ decay into the kaon related channels.

The low energy coupled channel equations studied in Refs. [19,20] deal only with the s-wave part of the $\bar{K} N$ interaction. A recent study of the p-wave $K^{-} N$ interaction at low energies within the context of chiral Lagrangians has been done in Ref. 25. Although the energies of the kaons in the $\phi$ decay are not large and hence the s-wave selfenergy plays the major role in the process, we nevertheless take the p-wave into account by including the excitation of the

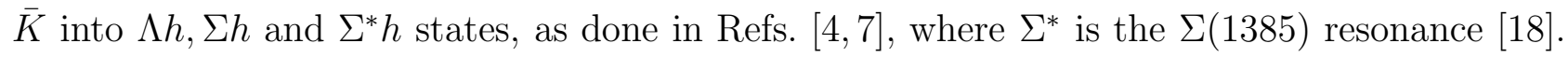
The p-wave selfenergy plays a role in the decay of the $\phi$ into $K^{+} Y h$ states, while the s-wave selfenergy contributes essentially to the decay into $K^{+} \Sigma \pi h$ states.

In the present work we use the s-wave $K^{-}$selfenergy from Ref. [11] and implement the p-wave selfenergy due to $\Lambda h, \Sigma h$ states, as also done in Ref. [11]. As just mentioned, we also include here the coupling of the $\bar{K}$ into $\Sigma^{*} h$ excitations. Its addition in the kaon loops in the selfconsistent calculation of the s-wave $K^{-}$selfenergy barely modifies the results of Ref. [11]. We will see, however, that as an extra contribution to the p-wave selfenergy it induces an increase in the $\phi$ width, since it incorporates the $\phi \rightarrow K^{+} \Sigma^{*} h$ decay channel.

In order to evaluate the $\phi$ selfenergy in the medium we write the $K^{+}$propagator as in eq. (8) using explicitly the $K^{+}$selfenergy of eq. (11). For the $K^{-}$propagator we use instead the Lehmann representation of eq. (9) and thus, following the same steps that led us to eq. (7), we obtain a $\phi$ width in the medium given by

$$
\begin{aligned}
\Gamma_{\phi}\left(P^{0}, \rho\right) & =\frac{1}{M_{\phi}} \int_{0}^{\omega_{\max }^{(+)}} d \omega S_{K^{-}}\left(\omega, q^{(+)}, \rho\right) \frac{1}{3 \pi} g_{\phi}^{2} q^{(+) 3} \\
& +\frac{1}{M_{\phi}} \int_{0}^{\omega_{\max }^{(0)}} d \omega S_{\bar{K}^{0}}\left(\omega, q^{(0)}, \rho\right) \frac{1}{3 \pi} g_{\phi}^{2} q^{(0) 3}
\end{aligned}
$$

where

$$
\begin{gathered}
q^{(+)}=\sqrt{\left(P^{0}-\omega\right)^{2}-\Pi_{K^{+}}-m_{K^{+}}^{2}}, \quad \omega_{\max }^{(+)}=P^{0}-\sqrt{m_{K^{+}}^{2}+\Pi_{K^{+}}} \\
q^{(0)}=\sqrt{\left(P^{0}-\omega\right)^{2}-\Pi_{K^{0}}-m_{K^{0}}^{2}}, \quad \omega_{\max }^{(0)}=P^{0}-\sqrt{m_{K^{0}}^{2}+\Pi_{K^{0}}}
\end{gathered}
$$


The vertex $\bar{K} N Y$ for an incoming kaon of momentum $\vec{k}$ is given by

$$
V_{\bar{K} N Y}=\widetilde{V}_{\bar{K} N Y} \vec{\sigma} \cdot \vec{k}=\left[\alpha \frac{D+F}{2 f}+\beta \frac{D-F}{2 f}\right] \vec{\sigma} \cdot \vec{k}
$$

where $\alpha$ and $\beta$ are given in Table 1 and $D+F=g_{A}=1.257, D-F=0.33$. We take $f=1.15 f_{\pi}$ with $f_{\pi}=93 \mathrm{MeV}$, a value which lies in between the pion and kaon weak decay constants and that was chosen in the model of Ref. [20] to optimize the position of the $\Lambda(1405)$ resonance.

Table 1: Coefficients for the $\bar{K} N Y$ couplings of eq. (14)

\begin{tabular}{c|cccccc} 
& $K^{-} p \rightarrow \Lambda$ & $K^{-} p \rightarrow \Sigma^{0}$ & $K^{-} n \rightarrow \Sigma^{-}$ & $\bar{K}^{0} n \rightarrow \Lambda$ & $\bar{K}^{0} n \rightarrow \Sigma^{0}$ & $\bar{K}^{0} p \rightarrow \Sigma^{+}$ \\
\hline$\alpha$ & $-\frac{2}{\sqrt{3}}$ & 0 & 0 & $-\frac{2}{\sqrt{3}}$ & 0 & 0 \\
$\beta$ & $\frac{1}{\sqrt{3}}$ & 1 & $\sqrt{2}$ & $\frac{1}{\sqrt{3}}$ & -1 & $\sqrt{2}$
\end{tabular}

Similarly, the $\bar{K} N \Sigma^{*}$ vertex is given by

$$
V_{\bar{K} N \Sigma^{*}}=\widetilde{V}_{\bar{K} N \Sigma^{*}} \vec{S}^{\dagger} \cdot \vec{k}=\frac{g_{\Sigma^{*}}}{2 M} A \vec{S}^{\dagger} \cdot \vec{k}
$$

where $\vec{S}^{\dagger}$ is the spin transition operator from spin $1 / 2$ to spin $3 / 2$. The coefficient A is given in Table 2 and the coupling $g_{\Sigma^{*}} / 2 M$ is evaluated here by first using the SU(6) quark model to relate the $\pi N N$ coupling to the $\pi N \Delta$ one and then using $\mathrm{SU}(3)$ symmetry to relate the $\pi N \Delta$ coupling to the $\bar{K} N \Sigma^{*}$ one, since the $\Sigma^{*}$ belongs to the $\mathrm{SU}(3)$ decouplet of the $\Delta$. We obtain:

$$
\frac{g_{\Sigma^{*}}}{2 M}=\frac{2 \sqrt{6}}{5} \frac{D+F}{2 f} .
$$

There is hence a small diversion of our approach here with respect to the one of Ref. 幽, where SU(3) arguments are used and, consequently, a different coupling was obtained. We note that with the coupling used in the present work one gets a good reproduction of the data for the decay of the $\Sigma^{*}$ into $\Lambda \pi$ and $\Sigma \pi$ final statesx, when the the value of $f$ is taken as $f_{\pi}$.

Table 2: Coefficient for the $\bar{K} N \Sigma^{*}$ couplings of eq. (15)

\begin{tabular}{c|cccc} 
& $K^{-} p \rightarrow \Sigma^{* 0}$ & $K^{-} n \rightarrow \Sigma^{*-}$ & $\bar{K}^{0} p \rightarrow \Sigma^{*+}$ & $\bar{K}^{0} n \rightarrow \Sigma^{* 0}$ \\
\hline$A$ & $-\frac{1}{\sqrt{2}}$ & -1 & -1 & $\frac{1}{\sqrt{2}}$
\end{tabular}

The p-wave $\bar{K}$ selfenergy in symmetric nuclear matter can then be written as

$$
\begin{aligned}
\Pi_{\bar{K}}^{(p)}\left(q^{0}, \vec{q}, \rho\right) & =\widetilde{\Pi}_{\bar{K}}(q, \rho) \vec{q}^{2} \\
& =\frac{1}{2} \widetilde{V}_{K^{-} p \Lambda}^{2} f_{\Lambda}^{2} \vec{q}^{2} U_{\Lambda}\left(q^{0}, \vec{q}, \rho\right) \\
& +\frac{3}{2} \widetilde{V}_{K^{-} p \Sigma^{0}}^{2} f_{\Sigma^{2}}^{2} \vec{q}^{2} U_{\Sigma}\left(q^{0}, \vec{q}, \rho\right) \\
& +\frac{1}{2} \widetilde{V}_{K^{-} p \Sigma^{* 0}}^{2} f_{\Sigma^{*}}^{2} \vec{q}^{2} U_{\Sigma^{*}}\left(q^{0}, \vec{q}, \rho\right)
\end{aligned}
$$


where the Lindhard function $U_{Y}(q)\left(Y=\Lambda, \Sigma\right.$ or $\left.\Sigma^{*}\right)$ is given by

$$
\begin{aligned}
\operatorname{Re} U_{Y}\left(q^{0}, \vec{q}, \rho\right) & =\frac{3}{2} \rho \frac{M_{Y}}{q p_{F}}\left\{z+\frac{1}{2}\left(1-z^{2}\right) \ln \frac{|z+1|}{|z-1|}\right\} \\
\operatorname{Im} U_{Y}\left(q^{0}, \vec{q}, \rho\right) & =-\pi \frac{3}{4} \rho \frac{M_{Y}}{q p_{F}}\left\{\left(1-z^{2}\right) \theta(1-|z|)\right\} \\
z & =\left(q^{0}-\frac{q^{2}}{2 M_{Y}}-\left(M_{Y}-M\right)\right) \frac{M_{Y}}{q p_{F}},
\end{aligned}
$$

with $\rho$ the nuclear matter density, $p_{F}$ the Fermi momentum and $f_{\Lambda}=\left(1-q^{0} / 2 M_{\Lambda}\right), f_{\Sigma}=$ $\left(1-q^{0} / 2 M_{\Sigma}\right), f_{\Sigma^{*}}=\left(1-q^{0} / M_{\Sigma^{*}}\right)$ relativistic recoil vertex corrections [11.

As in Ref. [7], we also incorporate a form-factor at the kaon-baryon vertices of dipole type, $\left[\Lambda^{2} /\left(\Lambda^{2}-q^{2}\right)\right]^{2}$, with $\Lambda=1.05 \mathrm{GeV}$.

Inserting the p-wave $K^{-}$selfenergy at the lowest order in the nuclear density accounts for the contribution to the in medium $\phi$ selfenergy depicted in diagram a) of Fig. 2. However, it was already noticed in Refs. [1,2] that, when dealing with gauge vector mesons, there are other vertex corrections associated to these diagrams which are required by the gauge invariance of the model. These diagrams are depicted in Figs. 2 b), c) and d). These terms appear from a contact $\phi K N Y$ term, similar to the Kroll Ruderman term for photons, and they can be evaluated systematically by substituting $e Q A_{\mu}$ by $g V_{\mu} / 2$ in the chiral Lagrangians, as done in Ref. [4], with $g=\frac{\sqrt{2}}{3} g_{\phi}$, where an extra minus sign is already incorporated in the Lagrangian of eq. (1). We thus obtain the following vertex function for these contact terms

$$
\begin{aligned}
V_{\phi K N Y} & =g_{\phi} \widetilde{V}_{\bar{K} N Y} \vec{\sigma} \cdot \vec{\epsilon}(\phi) ; \quad Y=\Lambda, \Sigma \\
V_{\phi K N \Sigma^{*}} & =g_{\phi} \widetilde{V}_{\bar{K} N \Sigma^{*}} \vec{S}^{\dagger} \cdot \vec{\epsilon}(\phi) .
\end{aligned}
$$

The contribution of the vertex corrections can be easily evaluated. We can see that the addition of all the one-baryon loop diagrams in Fig. 2 just replaces the contribution of the one in Fig. 2a), $D(q) \widetilde{\Pi}(q) \vec{q}^{2} D(q)$, by

$$
D(q) \widetilde{\Pi}(q) \vec{q}^{2} D(q)+\frac{1}{2} \widetilde{\Pi}(q) D(q)+\frac{1}{2} D(q) \widetilde{\Pi}(q)+\frac{3}{4} \frac{\widetilde{\Pi}(q)}{\vec{q}^{2}} .
$$

One simplifying step forward is possible at this point since we are interested in the imaginary part of the selfenergy. By means of Cutkosky rules one knows that the contributions to the imaginary part are obtained when placing either the $K^{+} K^{-}$or the $K^{+} Y h$ of the intermediate states on shell. In both cases the $K^{+}$appears on shell, which means that we can substitute $P^{0}-q^{0}$ by $\omega(q)$, hence $q^{0}=P^{0}-\omega(q)$, in eq. (20). It is then easy to see that the sum of all diagrams in Fig. 2 is equivalent to considering only diagram a) but with the following substitution for the kaon p-wave selfenergy

$$
\widetilde{\Pi}(q) \vec{q}^{2} \rightarrow \widetilde{\Pi}(q)\left[\left(P^{0}-\omega(q)\right)^{2}-m_{K}^{2}\right]\left\{1+\frac{3}{4} \frac{\left[\left(P^{0}-\omega(q)\right)^{2}-\vec{q}^{2}-m_{K}^{2}\right]^{2}}{\vec{q}^{2}\left[\left(P^{0}-\omega(q)\right)^{2}-m_{K}^{2}\right]}\right\} .
$$




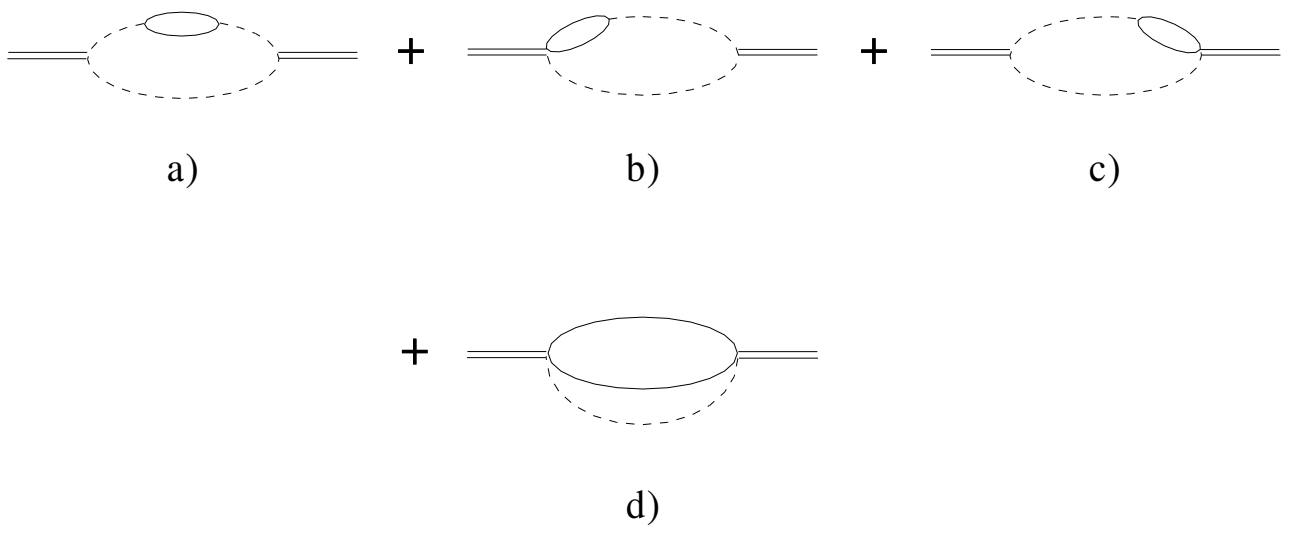

Figure 2: Selfenergy diagrams at first order in the nuclear density contributing to the decay of the $\phi$ meson in the medium.

The arguments given above apply to just the p-wave diagrams at lowest order in the density, i.e. those displayed in Fig. 2. In the actual calculation, however, we simply replace the p-wave selfenergy by means of eq. (21) in the $K^{-}$propagator which is used to evaluate the $\phi$ decay width in the medium, hence generalizing the correction of eq. (21) to higher orders of the density. This approximation is well justified because when we pick up the imaginary part from the two kaon cut the momenta involved are small and the p-wave plays a minor role. On the other hand, the p-wave part becomes relevant when we pick up the $K^{+} Y h$ excitation, in which case there is plenty of phase space available and the momenta involved are large. In this case, however, the $\bar{K}$ is far off shell and an additional particle-hole insertion in the $\bar{K}$ selfenergy, i.e. a higher order in the density, does not modify much the $\bar{K}$ propagator.

The use of the technique explained above simplifies the analytical structure of the integrand of the $\phi$ selfenergy and allows one to use the same formalism that leads to eq. (12), where there are only $\mathrm{p}($ and $\mathrm{s}$ )-wave selfenergy insertions in the propagators and no vertex corrections.

\section{Results}

In Fig. 3 we show the different contributions to the $\phi$ width as a function of the $\phi$ energy. The dotted line represents the contribution to the $\phi$ width when the $\bar{K}$ is dressed with only the s-wave selfenergy (the $K$ is dressed with the repulsive selfenergy of eq. (11) in all these curves). We can already see an appreciable increase of the width with respect to the free value of about $4 \mathrm{MeV}$. This is due on the one hand to the fact that the $\bar{K}$ feels an attraction of bigger strength than the repulsion felt by the $K$, hence the phase space for $K \bar{K}$ decay increases. On the other hand, the $\bar{K}$ in the nucleus decays in s-wave into the channels $\pi \Sigma h$ and $\pi \Lambda h$ and, therefore, 
the incorporation of the $\bar{K}$ s-wave selfenergy in the evaluation of the $\phi$ selfenergy automatically accounts for the $\phi \rightarrow K \pi \Sigma h$ and $\phi \rightarrow K \pi \Lambda h$ channels or, conversely, the nucleon induced $\phi$ decay reactions $\phi N \rightarrow K \pi \Sigma$ and $\phi N \rightarrow K \pi \Lambda$.

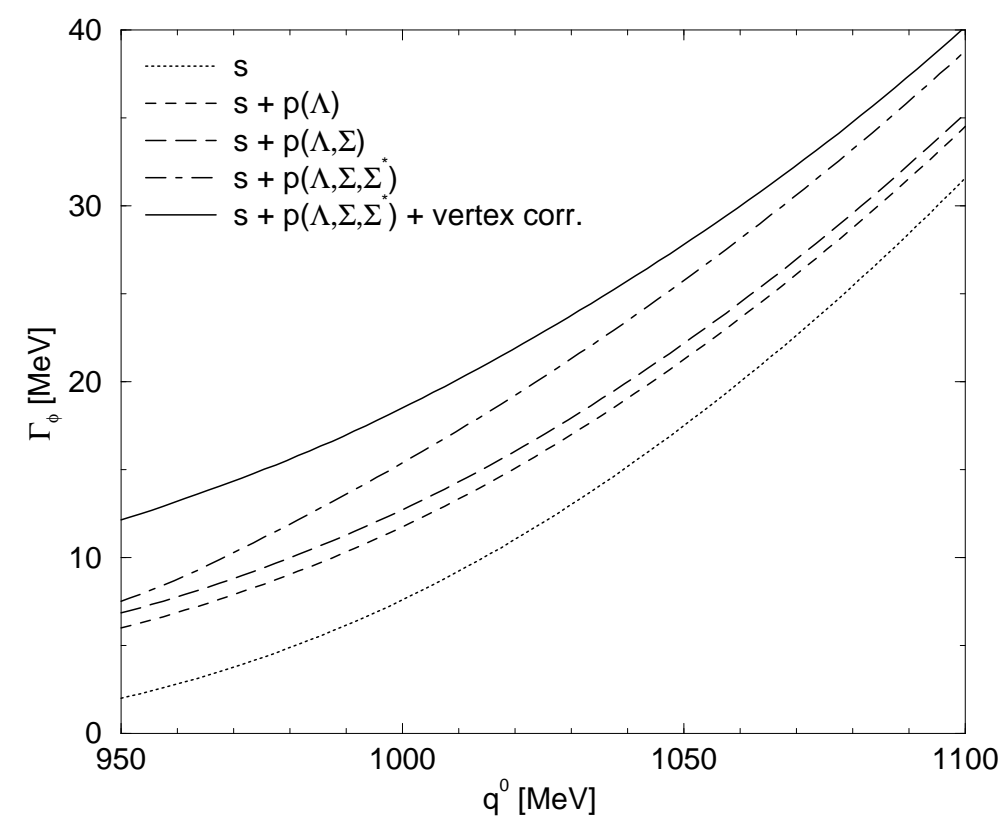

Figure 3: Width of the $\phi$ meson in nuclear matter at density $\rho=\rho_{0}$ as a function of the $\phi$ energy.

A next step in the figure incorporates the contribution of decay channels related to the p-wave $\bar{K}$ selfenergy through $Y h$ excitations, with $Y=\Lambda$ (short dashed curve), $Y=\Lambda, \Sigma$ (longdashed curve) and $Y=\Lambda, \Sigma, \Sigma^{*}$ (dot-dashed curve). These correspond to the reactions $\phi N \rightarrow$ $K \Lambda, K \Sigma, K \Sigma^{*}$. We can see that the largest contribution corresponds to the $\Lambda h$ excitation, as already noted in Ref. [7], since it has a larger phase space and in addition the $\bar{K} N \Lambda$ coupling involves the $D+F$ combination, while the $\bar{K} N \Sigma$ one involves the $D-F$ combination, which is about a factor four smaller. The contribution of the $\Sigma^{*}$ is comparatively larger than the $\Sigma$ one, due to the larger coupling, but still smaller than that of the $\Lambda$ due to the reduced phase space.

Finally, the solid line in Fig. 3 shows the results obtained when the vertex corrections are also incorporated. They increase the width of the $\phi$ by an additional $3 \mathrm{MeV}$ at the $\phi$ mass, $M_{\phi}=1019.413 \mathrm{MeV}$.

The final width of the $\phi$ meson at the $\phi$ mass in symmetric nuclear matter of density $\rho=\rho_{0}$ amounts to $22 \mathrm{MeV}$. All the different decay channels contributing to the final width increase smoothly with energy as a consequence of the increasingly larger available phase space.

Although not shown in the figure, the effect of the relativistic recoil corrections (factors $f_{Y}$ in eq. (18) ) amounts to a reduction of about $5 \mathrm{MeV}$, also at the $\phi$ mass. If the dipole form factor is omitted the $\phi$ width raises to about $30 \mathrm{MeV}$. If, on the other hand, one uses a monopole 
factor of the type $\Lambda^{2} /\left(\Lambda^{2}-q^{2}\right)$, with $\Lambda=1.3 \mathrm{GeV}$, as commonly used in phenomenological studies of the hyperon-nucleon interaction, the $\phi$ width turns out to be around $26 \mathrm{MeV}$.

In Fig. 1 we show the total width of the $\phi$ as a function of the $\phi$ energy for three different densities. As one can see from the figure, the medium effects are not proportional to the density. The observed density dependence also differs for the different energies of the $\phi$. We have checked that this highly nonlinear density behavior of the width comes mainly from the s-wave part of the $\bar{K}$ selfenergy, as already noted in Ref. [7]. At $\rho=\rho_{0}$ this s-wave contribution to the width is reduced by a factor 2.5 with respect to a linear extrapolation from low densities. The p-wave contribution is more moderately density dependent, but there is still a reduction of about $30 \%$ at $\rho=\rho_{0}$ with respect to the linear extrapolation from low densities, as also estimated in Ref. [7]. In the present work we take into account the full density dependence of the p-wave contribution, which in Refs. [4, [7] was only considered at the lowest order in the density.

The analysis done here about the density dependence and the p-wave contributions can be used to support the arguments given at the end of Sect. 2 justifying the use of the prescription of eq. (21) to incorporate the p-wave vertex corrections. We have carried out a different calculation in which $\vec{q}^{2}$ is substituted in the $\phi$ vertices by the term on the right in expression (21) divided by $\widetilde{\Pi}(q)$. This new prescription is equivalent to the one used before in the case of just one particle-hole excitation in the p-wave part (it should not be used for the s-wave part where there are no vertex corrections). However, this new prescription does not modify the p-wave selfenergy at higher orders in the density, a feature we would like to have in our calculation. The differences between the two prescriptions are of the order of $2 \%$ at $\rho=\rho_{0}$, which gives us an idea of the accuracy of the calculation.

The width obtained in the present work is about a factor of two smaller than that obtained in Refs. [4, []. Had we used $f=f_{\pi}$ the width would have raised only up to $25 \mathrm{MeV}$. One might think that the differences with the result in Ref. [7] should be attributed to the fact that the latter work relied on a $\bar{K}$ selfenergy that was not selfconsistently evaluated [9]. Their in-medium $\bar{K} N$ amplitude included basically the Pauli-blocking effects on the nucleons in the intermediate $\bar{K} N$ loops. As a result, the $\bar{K}$ strength at $\rho_{0}$ presents a quite narrow peak [9], located $100 \mathrm{MeV}$ below the kaon mass. In contrast, when the attractive and complex $\bar{K}$ selfenergy is incorporated selfconsistently in the in-medium scattering equation, as done in Refs. [10, 11], the peak of the $\bar{K}$ spectral function moves to energies closer to the free $\bar{K}$ mass, which should result in a drastic reduction of the $\phi$ width. However, when we use the $\bar{K}$ selfenergy which only incorporates the Pauli-blocking medium effects, an approximation which is also discussed in Ref. [11], the width is only $7 \%$ larger than that obtained using the selfconsistent $\bar{K}$ dressing. The reason is that the extra widening of the selfconsistent $\bar{K}$ spectral function weakens the reduction caused by 


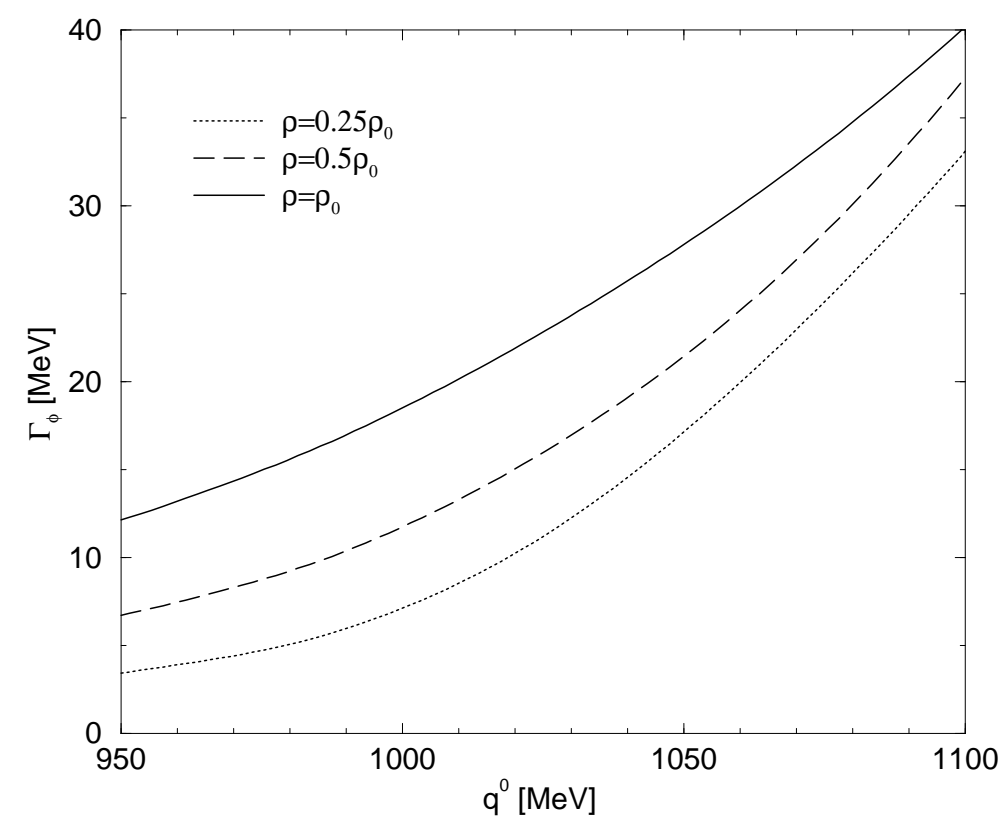

Figure 4: Width of the $\phi$ meson in nuclear matter at three densities $\left(\rho=0.25 \rho_{0}, 0.5 \rho_{0}, \rho_{0}\right)$ as a function of the $\phi$ energy.

the larger mass of the $\bar{K}$ in the medium. Another factor which contributes to obtaining a smaller result than that found in Refs. [4, [] is the inclusion in the present work of the p-wave $\bar{K}$ selfenergy to all orders in the $\bar{K}$ propagator, while only the lowest order term in the density was considered in Refs. [4, [].

The tests done in this section give us an idea of the theoretical uncertainties of the present model, which we can estimate in a band between $22-28 \mathrm{MeV}$ for the $\phi$ width at normal nuclear matter density. Yet, when these numbers are compared with the free width of $4.4 \mathrm{MeV}$, the important message, shared with Ref. [7], is that the width of the $\phi$ is appreciably enhanced in nuclei by nearly an order of magnitude.

Finally, the real and imaginary parts of the $\phi$ propagator, normalized such that the later

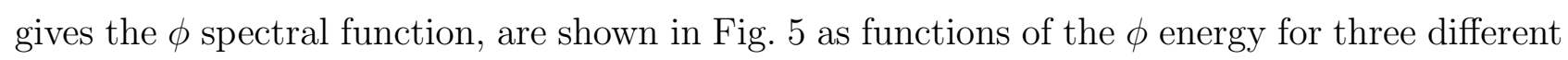
densities. The mass of the $\phi$ meson in the medium has been taken to be the same as the free one, an assumption that relies upon the results of Ref. [4] where only a small change of about $1 \%$ was found. The novel elements introduced here in the kaon spectral function should not change this result qualitatively.

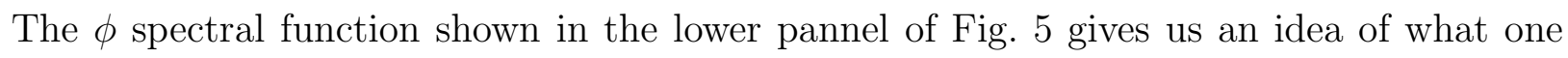
might expect for, let us say, the invariant mass of $K \bar{K}$ distributions in nuclei around the $\phi$ mass in $\phi$ production experiments, like $\gamma$-nucleus or $\pi$-nucleus collisions. These reactions could be easily done in experimental facilities like Spring8/Osaka or GSI, where programs to produce $\phi$ mesons in the elementary reactions are already considered. Certainly, the results obtained here, 
extrapolated if needed at higher densities, should also be of much use in analyses of dilepton production in heavy ion collisions around the $\phi$ energy, which is actually one of the regions studied experimentally.

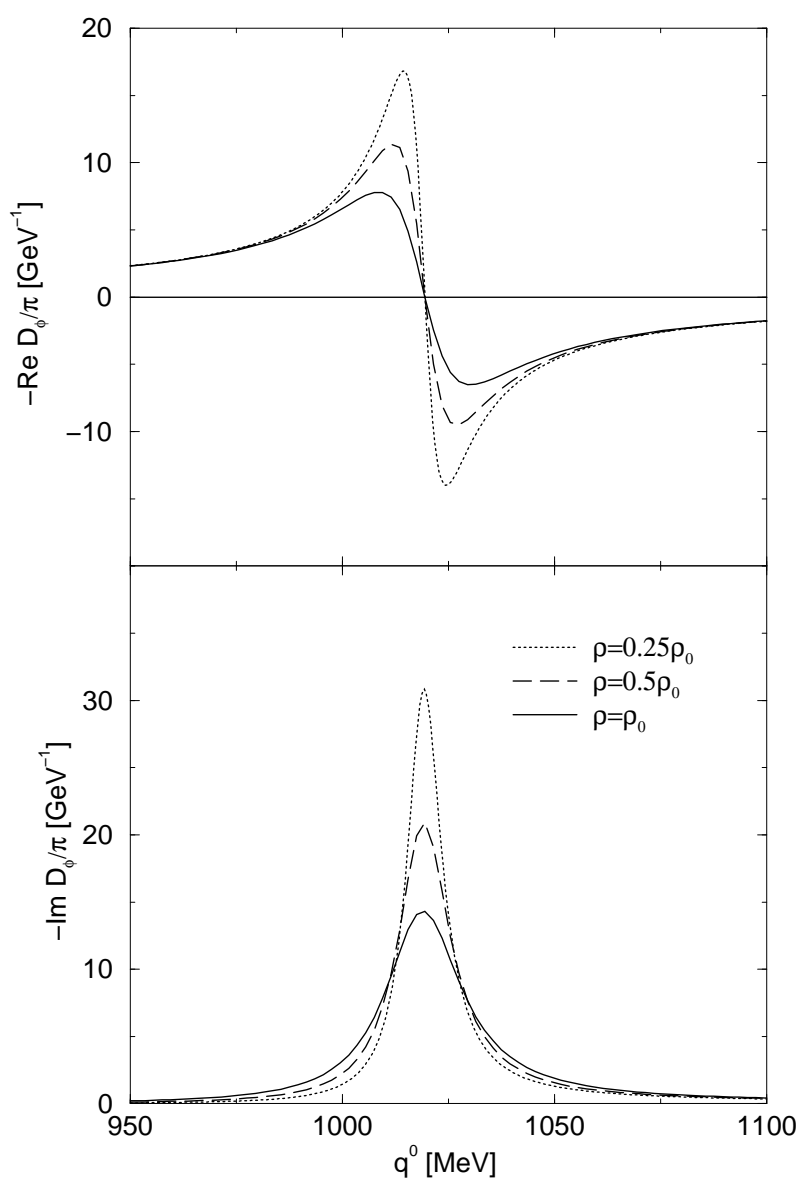

Figure 5: Real and Imaginary parts of the $\phi$ meson propagator in nuclear matter at three densities $\left(\rho=0.25 \rho_{0}, 0.5 \rho_{0}, \rho_{0}\right)$ as functions of the $\phi$ energy.

\section{Conclusions}

We have studied the different mechanisms of $\phi$ decay in a nuclear medium. We rely upon the OZI rule which makes the direct coupling of the $\phi$ to nucleons or nonstrange mesons small, and thus the $\phi$ decay is still driven by the $K \bar{K}$ or related channels in the medium. The $\phi$ width in the medium is hence obtained from previously developed models for the interaction of kaons in nuclei, which have been tested in $K^{-}$atoms recently. The gauge nature of the $\phi$ as a vector field also introduces some vertex corrections which lead to a moderate increase of the $\phi$ decay width.

We have evaluated this width as a function of the energy of the $\phi$ and the nuclear density 
of the medium and have separated the contribution of the different reaction channels, which can eventually be investigated in some experiments for $\phi$ production in nuclei. The relevance of these findings for the dressing of kaons in nuclei and its relationship to the eventual kaon condensation in dense matter, as well as the implications for the running experiments of dilepton production in heavy ion collisions, should stimulate the performance of $\phi$ production in nuclei with some particle nucleus collisions which could serve us to test the ideas and results obtained in the present work.

\section{Acknowledgements}

We would like to thank B. Friman for discussions . This work has been partially supported by the DGICYT contract numbers PB98-1247 and PB96-0753, and by the EEC-TMR Program, EURODAPHNE, under contract number CT98-0169.

\section{References}

[1] M. Herrmann, B.L. Friman and W. Norenberg, Nucl. Phys. A560 (1993) 411

[2] G. Chanfray and P. Schuck, Nucl. Phys. A555 (1993) 329

[3] R. Rapp, G. Chanfray and J. Wambach, Nucl. Phys. A617 (1997) 472

[4] F. Klingl, N. Kaiser and W. Weise, Nucl. Phys. A624 (1997) 527

[5] J. Friese, Prog. Part. Nucl. Phys. 42 (1999) 135

[6] G. Agakishiev et al., Phys. Lett. B422 (1998) 405

[7] F. Klingl, T. Waas and W. Weise, Phys. Lett. B431 (1998) 254

[8] V. Koch, Phys. Lett. B337 (1994) 7

[9] T. Waas and W. Weise, Nucl. Phys. A625 (1997) 287

[10] M. Lutz, Phys. Lett. B426 (1998) 12

[11] A. Ramos and E. Oset, Nucl. Phys. A, in print. nucl-th/9906016

[12] D.B. Kaplan and A.E. Nelson, Phys. Lett. B175 (1986) 57

[13] C.J. Batty, Nucl. Phys. A372 (1981) 418 
[14] C.J. Batty, E. Friedman and A. Gal, Phys. Rep. 287 (1997) 385

[15] A. Baca, C. García-Recio and J. Nieves, Nucl. Phys. A, in print. nucl-th/0001060

[16] S. Hirenzaki, Y. Okumura, H. Toki, E. Oset and A. Ramos, Phys. Rev. C61 (2000) 055205

[17] G. Ecker, J. Gasser, A. Pich and E. de Rafael, Nucl. Phys. B321 (1989) 311

[18] C. Caso et al., The European Physical Journal C3 (1998) 1

[19] N. Kaiser, P.B. Siegel, and W. Weise, Nucl. Phys. A594 (1995) 325; N. Kaiser, T. Waas and W. Weise, Nucl. Phys. A612 (1997) 297

[20] E. Oset and A. Ramos, Nucl. Phys. A635 (1998) 99

[21] R.H. Dalitz and S.F. Tuan, Ann. Phys. (N.Y.) 10 (1960) 307; R.H. Dalitz, T.C. Wong and G. Rajasekaran, Phys. Rev. 153 (1967) 1617

[22] A.D. Martin, Nucl. Phys. B179 (1981) 33

[23] P.B. Siegel and B. Saghai, Phys. Rev. C52 (1995) 392

[24] J.A. Oller, E. Oset and A. Ramos, Prog. Part. Nucl. Phys. 45, in print. hep-th/0002193

[25] J. Caro, N. Kaiser, S. Wetzel and W. Weise, Nucl. Phys. A in print, nucl-th/9912035 